\title{
Effects of nuclear structure on average angular momentum in subbarrier fusion
}

\author{
A.B. Balantekin, J.R. Bennett \\ Physics Department, University of Wisconsin, \\ Madison, Wisconsin, 53706 USA \\ S. Kuyucak \\ Department of Theoretical Physics, \\ Research School of Physical Sciences, \\ Australian National University, Canberra, ACT 0200, Australia
}

\begin{abstract}
We investigate the effects of nuclear quadrupole and hexadecapole couplings on the average angular momentum in sub-barrier fusion reactions. This quantity could provide a probe for nuclear shapes, distinguishing between prolate vs. oblate quadrupole and positive vs. negative hexadecapole couplings. We describe the data in the $\mathrm{O}+$ $\mathrm{Sm}$ system and discuss heavier systems where shape effects become more pronounced.
\end{abstract}

Address correspondence to A. B. Balantekin.

E-mail: baha@wisnud.physics.wisc.edu 
Enhancement of subbarrier fusion cross sections in medium and heavy mass nuclei due to coupling of internal degrees of freedom to the relative motion is a well established phenomenon (see Refs. [1] and [2] for reviews). Because subbarrier fusion is sensitive to details of nuclear structure, one can consider using it as a technique to measure structure information which is complementary to other methods. As is well known, the so-called "distribution of barriers" [3], determined from the quantity

$$
D(E) \equiv \frac{d^{2}}{d E^{2}}(E \sigma(E))
$$

has a shape which distinguishes among different types of nuclear structure, for example prolate versus oblate, rotational versus vibrational, etc. In this paper, we point out that the average angular momentum transferred to the compound nucleus, $\langle\ell\rangle$, is also sensitive to different kinds of nuclear shapes. Also, we emphasize that partial cross section distributions $\sigma_{\ell}$ having different mean values $\langle\ell\rangle$ can give the same total cross section $\sigma$. Therefore, it is important to consistently describe both the fusion cross section and the average angular momentum data.

For several systems, a "bump" in $\langle\ell\rangle$, a sudden rise in the angular momentum as the energy increases toward the barrier from below, followed by a flattening off above the barrier, has been observed [4]. We will show that the "bump" in the $\langle\ell\rangle$-distribution happens for prolate systems but not for oblate ones. Other authors have previously noted this difference in the $\ell$ distributions of a prolate and an oblate nucleus [5]. We emphasize here that the different distributions are characteristic of the two shapes. Also, we show that the addition of a positive or negative hexadecapole moment can add or subtract, respectively, several units of angular momentum from the bump. Hence, the bump in $\langle\ell\rangle$, or its absence, can be a signature for various nuclear deformations. In systems with light projectiles such as ${ }^{16} \mathrm{O}$, observing such signatures requires measuring $\langle\ell\rangle$ to within one unit of angular momentum which may not be easy. Using heavier projectiles, e.g. ${ }^{40} \mathrm{Ca}$, magnifies the coupling effects several times, which offers an easier identification of nuclear shape effects.

We calculate the total fusion cross section using the partial wave expansion in the barrier penetration picture. The penetration probabilities for the different partial waves are evaluated numerically using a uniform WKB approximation, valid for energies both above and below the barrier [6] 


$$
T_{\ell}(E)=\sum_{i} \omega_{i}\left[1+\exp \left(2 \sqrt{\frac{2 \mu}{\hbar^{2}}} \int_{r_{1}}^{r_{2}} d r\left(V\left(r, \lambda_{i}\right)-E\right)^{\frac{1}{2}}\right)\right]^{-1} .
$$

Here, $r_{1}$ and $r_{2}$ are the classical turning points of the motion, and $\omega_{i}$ refers to the weight in the eigenchannel $i$ with corresponding eigenvalue $\lambda_{i}$. This result is the familiar representation of the subbarrier fusion cross section as a weighted average of cross sections due to tunnelling through a set of potential barriers. In the geometrical description of the nucleus, the barriers are determined by averaging over fixed orientations of a deformed nucleus [7] or over zero-point vibrations of the surface of a vibrational nucleus 8]. Alternatively, in the interacting boson model description of nuclei[9], the barriers are determined as described in Ref. [10] from the eigenvalues $\lambda_{i}$ of the coupling operator

$$
\hat{O}=v_{2} \frac{Q}{\left\langle 2_{1}|| Q \| 0_{1}\right\rangle} \cdot Y^{(2)}(\hat{\mathbf{r}})+v_{4} \frac{H}{\left\langle 4_{1}|| H|| 0_{1}\right\rangle} \cdot Y^{(4)}(\hat{\mathbf{r}}),
$$

where $Q=\left[s^{\dagger} \tilde{d}+d^{\dagger} s\right]^{(2)}+\chi\left[d^{\dagger} \tilde{d}\right]^{(2)}$ and $H=\left[d^{\dagger} \tilde{d}\right]^{(4)}$ are the quadrupole and hexadecapole transition operators, respectively. Here, the operators $s^{\dagger}(s)$ and $d^{\dagger}(d)$ create (annihilate) bosons of angular momentum zero and two, respectively, representing correlated pairs of valence nucleons. The parameters $v_{2}$ and $v_{4}$ describe the quadrupole and hexadecapole couplings and are roughly proportional to the E2 and E4 excitation strengths in the target nucleus. In the IBM description, the weights $\omega_{i}$ are given by the overlaps of the eigenstates of the coupling operator $\hat{O}$ with the ground state of the target nucleus, which we project from an intrinsic state as follows

$$
|J=0, M=0\rangle=\frac{1}{\mathcal{N}} \int d \theta \sin \theta R(\theta)\left(b^{\dagger}\right)^{N}|0\rangle,
$$

where $\mathcal{N}$ is a normalization factor and we also introduced the intrinsic boson operator, $b^{\dagger}=x_{0} s^{\dagger}+x_{2} d_{0}^{\dagger}$. The target wave functions are represented by the mean fields $x_{0}$ and $x_{2}$ for the $s$ and $d$ bosons which are determined from a given IBM Hamiltonian (which fits the low-lying spectroscopic data) by variational techniques [11]. The parameter $\chi$ is also determined from this method. We emphasize here the utility of the IBM description of collective nuclear structure in providing a description of rotational, vibrational and 
transitional nuclei within a unified theoretical framework. This quality becomes especially useful in describing the transitional nuclei, such as ${ }^{150} \mathrm{Sm}$ (considered later in this paper), which are more difficult to handle in geometrical models. As has been emphasized by many authors [12, 13], the validity of expression (5) depends only on the usual adiabatic and rotating frame approximations which we have discussed previously [15].

For demonstrative purposes, we first consider a fictitious target nucleus with $A=170$ and $Z=70$, to which we assign various static deformations in order to isolate specific nuclear structure effects. In addition, we suppose for simplicity that the projectile is ${ }^{16} \mathrm{O}$ so that the parameters of the WoodsSaxon potential are fixed from our previous systematic study of subbarrier fusion cross sections and barrier distributions with that projectile[10]. Hence we take a simple global parameterization of the Woods-Saxon potential, taking in all cases $R_{2}=2.62 \mathrm{fm}, R_{1}=1.04 A_{1}^{1 / 3} \mathrm{fm}, V_{0}=67.5\left(A_{1} / 144\right)^{1 / 3} \mathrm{MeV}$, $a=1.22 \mathrm{fm}$.

In Fig. [1a], we show for a prolate-deformed nucleus the effects of positive and negative hexadecapole moments on $\langle\ell\rangle$. The solid curve has no hexadecapole moment $\left(v_{4}=0\right)$, the dashed one has a positive hexadecapole moment and the dotted curve has a negative one. The barrier for this system is at about $66 \mathrm{MeV}$. At energies near the barrier, the positive (negative) hexadecapole moments add (subtract) about one unit of angular momentum. This effect is due to constructive (destructive) interference between the quadrupole and hexadecapole couplings and was observed previously in experiments on ${ }^{16} \mathrm{O}+{ }^{154} \mathrm{Sm},{ }^{186} \mathrm{~W}$ subbarrier fusion [14 and in our systematic study of subbarrier fusion cross sections [10. The effects of hexadecapole moments on angular momentum transfer in subbarrier fusion reactions have not been considered previously. Fig. [1b] compares the angular momentum transfer as a function of energy for a prolate target with that for an oblate target of the same deformation. For reference, we also show the calculated average angular momentum for a nucleus with no deformation. In comparing Figs. 1a and $1 b$, we see that including hexadecapole coupling changes $\langle\ell\rangle$ in the vicinity of the barrier energy by about $25 \%$ of the increase due to including quadrupole coupling only. The prolate target displays the "bump" in the $\ell$ - distribution. The oblate nucleus shows an increased angular momentum from the spherical nucleus at energies near the barrier, but does not have the bump.

In Figs. [2a-2d], we show the results of using our global nuclear potential 
to calculate the fusion cross section and average angular momentum for a series of four isotopes, ${ }^{148,150,152,154} \mathrm{Sm}$, for which experimental data on the fusion cross section are available and average angular momenta are either measured or deduced [16, 17, 18, 19, 20, 21, 22]. The quadrupole and hexadecapole coupling strengths, $v_{2}$ and $v_{4}$, are determined from a simultaneous fit to all the available data. These parameters and the mean fields $x_{0}$ and $x_{2}$ for all of the calculations are listed in Table I. In our previous analysis of fusion cross sections and barrier distributions [10 for the nuclei ${ }^{148} \mathrm{Sm}$ and ${ }^{154} \mathrm{Sm}$, we obtained values for these coupling parameters which were mostly consistent with the known E2 and E4 matrix elements for those nuclei. In that study, we did not attempt to describe the data for ${ }^{150} \mathrm{Sm}$ and ${ }^{152} \mathrm{Sm}$ since barrier distributions were not available for those systems. In the present calculations, we have found that in order to explain the fusion cross section data it is necessary to assume quadrupole and hexadecapole couplings which do not change from nucleus to nucleus in a manner consistent with E2 and E4 matrix elements, or equivalently with the geometrical deformation parameters $\beta_{2}$ and $\beta_{4}$ as measured in Coulomb excitation experiments [23]. This behavior of the couplings in subbarrier fusion has been observed by other authors [24, 25]. In particular, in Coulomb excitation measurements, the nuclear deformations are found to decrease rapidly and suddenly in the region of the phase transition from rotational to vibrational nuclei around ${ }^{150} \mathrm{Sm}\left(\beta_{2}\left({ }^{152} \mathrm{Sm}\right)=0.29, \beta_{2}\left({ }^{148} \mathrm{Sm}\right)=0.14\right)$, whereas to describe the subbarrier fusion data these deformations must change much more slowly. This discrepancy between the deformations determined in the two different experiments suggests that the meaning of the couplings in subbarrier fusion is not necessarily the same as that suggested by a simple geometrical picture. Investigation of this point may require a description of subbarrier fusion in a deeper theoretical framework than the one we are using and so will be left to later studies.

In general one sees that the fusion cross sections can be described quite well by our global model. The angular momentum distributions are also reproduced well except for a systematic underestimation of the angular momentum at energies where the "bump" in the calculated distributions flattens off. The reason for this discrepancy may be due to the need for inclusion of couplings to other reaction channels or to some more basic shortcoming of our model of the fusion process.

Our purpose in this letter is to show how nuclear structure effects can 
affect the average angular momentum transferred to the compound nucleus in subbarrier fusion reactions. We have demonstrated the sorts of effects one can expect to observe and shown the validity of our model for reactions with heavy collective nuclei. In order to emphasize the usefulness of subbarrier fusion as a sensitive probe of nuclear structure, we would next like to calculate the expected angular momentum distributions for reactions with ${ }^{192} \mathrm{Os}$ and ${ }^{194} \mathrm{Pt}$. The former nucleus is known to be prolate, the latter to be oblate. These nuclei have never been studied in subbarrier fusion reactions, although experiments to measure the cross section and barrier distribution are currently being carried out at the Australian National University and the University of Washington. In Fig. [3a] we show the average angular momentum calculated using our global nuclear potential for the reactions ${ }^{16} \mathrm{O}+{ }^{192} \mathrm{Os},{ }^{194} \mathrm{Pt}$. The presence or absence of the bump near the barrier indicates the difference between prolate and oblate target. This effect can be enhanced, however, by using a heavier projectile nucleus so that the reduced mass of the system is larger. This dependence of the size of the bump on the reduced mass of the entrance channel has been demonstrated in measurements of the reactions ${ }^{16} \mathrm{O}+{ }^{166} \mathrm{Er}$ and ${ }^{28} \mathrm{Si}+{ }^{154} \mathrm{Sm}$ which lead to the same compound nucleus [2]. Hence in Fig. [3b] we show calculations for the angular momentum for the reactions ${ }^{40} \mathrm{Ca}+{ }^{192} \mathrm{Os},{ }^{194} \mathrm{Pt}$. In Fig. [3b] one can clearly see the bump for the prolate target and no bump for the oblate one. For these calculations with ${ }^{40} \mathrm{Ca}$ as the projectile, we used the same global Woods-Saxon potential as with the ${ }^{16} \mathrm{O}$ projectile, except that the nuclear potential strength was parameterized as $V_{0}=123.9\left(A_{1} / 144\right)^{1 / 3} \mathrm{MeV}$. Our systematic study of reactions involving ${ }^{16} \mathrm{O}$ projectiles gives us some confidence in predicting the results of other reactions with that nucleus. It is possible, however, that one must consider additional channels such as projectile excitation in reactions with heavier projectiles. Hence, we should emphasize that these calculations are intended only to give a qualitative estimate of the cross section and angular momentum to be expected for these reactions.

We have given a consistent description of subbarrier fusion cross section and angular momentum data for a series of reactions of ${ }^{16} \mathrm{O}$ with samarium isotopes. The nuclear structure of the target nuclei greatly influences both the cross sections and the angular momenta. We have demonstrated that measurement of the average angular momentum as a function of energy may be able to distinguish between target nuclei which are prolate (these show a bump near the barrier) and oblate (these show no bump). Nuclear hexade- 
capole moments also influence the angular momentum, though not as much as do quadrupole moments. We hope that our work will prompt further experimental investigation of angular momentum in subbarrier fusion, as this quantity provides an important constraint on models of fusion.

This research was supported in part by the U.S. National Science Foundation Grants No. PHY-9314131 and INT-9315876, in part by the Australian Research Council and in part by an exchange grant from the Department of Industry, Technology and Commerce of Australia. One of us (J.R.B.) would like to thank the members of the Department of Theoretical Physics at the Australian National University for their hospitality while this work was being carried out. 


\section{References}

[1] M. Beckerman, Rep. Prog. Phys. 51 (1988) 1047.

[2] R. Vandenbosch, Ann. Rev. Nuc. Part. Sci. 42 (1992) 447.

[3] N. Rowley, G.R. Satchler and P.H. Stelson, Phys. Lett. B 254 (1991) 25.

[4] S. Gil et al., Phys. Rev. Lett. 65 (1990) 3100.

[5] N. Rowley, J.R. Leigh, J.X. Wei and R. Lindsay, Phys. Lett. B 314 (1993) 179.

[6] D.M. Brink, Semi-classical Methods for Nucleus-Nucleus Scattering (Cambridge, 1985).

[7] D. M. Chase, L. Wilets and A. R. Edmonds, Phys. Rev. 110 (1958) 1080.

[8] H. Esbensen, Nucl. Phys. A 352 (1981) 147.

[9] F. Iachello and A. Arima, The Interacting Boson Model (Cambridge, 1987).

[10] A.B. Balantekin, J.R. Bennett, and S. Kuyucak, Phys. Rev. C 49 (1994) 1079 .

[11] S. Kuyucak and I. Morrison, Ann. Phys. (N.Y.) 181 (1988) 79.

[12] M. A. Nagarajan, A. B. Balantekin and N. Takigawa, Phys. Rev. C 34 (1986) 894.

[13] N. Rowley, Japanese J. Appl. Phys. Series 9 (1993) 218.

[14] R. C. Lemmon et al., Phys. Lett. B 316 (1993) 32.

[15] A.B. Balantekin, J.R. Bennett, A.J. DeWeerd and S. Kuyucak, Phys. Rev. C 46 (1992) 2019.

[16] R.G. Stokstad, Y. Eisen, S. Kaplanis, D. Pelte, U. Smilansky and I. Tserruya, Phys. Rev. C 21 (1980) 2427.

[17] J.X. Wei et al., Phys. Rev. Lett. 67 (1991) 3368. 
[18] J.R. Leigh et al., Phys. Rev. C 47 (1993) R437.

[19] J.R. Leigh et al., in: Proc. RIKEN workshop on Heavy-Ion Reactions with Neutron-Rich Beams (Japan, 1993).

[20] A. H. Wuosmaa et al., Phys. Lett. B 263 (1991) 23.

[21] J.D. Bierman, A.W. Charlop, D.J. Prindle, R. Vandenbosch and D. Ye, Phys. Rev. C 48 (1993) 319.

[22] C. V. K. Baba, Nucl. Phys. A 553 (1993) 719c.

[23] Nuclear Data Sheets 58 (1989) 93; Nuclear Data Sheets 59 (1990) 393.

[24] D.E. DiGregorio et al., Phys. Lett. B 176 (1986) 322.

[25] R.G. Stokstad and E.E. Gross, Phys. Rev. C 23 (1981) 281.

Table I

\begin{tabular}{lccccc}
\hline Nucleus & $x_{0}$ & $x_{2}$ & $\chi$ & $v_{2}$ & $v_{4}$ \\
\hline${ }^{148} \mathrm{Sm}$ & 0.92 & 0.39 & -1.15 & 0.18 & 0.0 \\
\hline${ }^{150} \mathrm{Sm}$ & 0.91 & 0.41 & -1.15 & 0.22 & 0.02 \\
${ }^{152} \mathrm{Sm}$ & 0.77 & 0.64 & -1.15 & 0.25 & 0.055 \\
\hline${ }^{154} \mathrm{Sm}$ & 0.74 & 0.67 & -1.15 & 0.26 & 0.06 \\
\hline${ }^{170} \mathrm{Yb}$ & 0.64 & 0.77 & -0.66 & - & - \\
\hline${ }^{192} \mathrm{Os}$ & 0.69 & 0.72 & -0.20 & 0.19 & -0.03 \\
\hline${ }^{194} \mathrm{Pt}$ & 0.69 & -0.72 & 0.15 & 0.17 & -0.03
\end{tabular}

Nuclear structure parameters for the nuclei which we consider. 


\section{Figure Captions}

FIG. 1. a) The effect of hexadecapole coupling on average angular momentum for a prolate target $\left(v_{2}=0.24\right)$. The dotted, dashed and solid lines are for $v_{4}=-0.033,0,+0.033$, respectively. b) Comparison of average angular momentum for prolate (solid line) and oblate (dashed line) targets and for no coupling (dotted line) There is no hexadecapole coupling $\left(v_{4}=0\right)$.

FIG. 2. a) Comparison of predicted fusion cross section and average angular momentum with the experimental data for the reaction ${ }^{16} \mathrm{O}+{ }^{148} \mathrm{Sm}$. The coupling parameters are given in Table I. b) Same as Fig. 2a but for ${ }^{150} \mathrm{Sm}$. c) Same as Fig. 2a but for ${ }^{152} \mathrm{Sm}$. d) Same as Fig. 2a but for ${ }^{154} \mathrm{Sm}$.

FIG. 3. a) Predicted fusion cross sections and average angular momenta for the reactions ${ }^{16} \mathrm{O}+{ }^{192} \mathrm{Os}$ (solid line) and ${ }^{16} \mathrm{O}+{ }^{194} \mathrm{Pt}$ (dashed line). b) Predicted fusion cross sections and average angular momenta for the reactions ${ }^{40} \mathrm{Ca}+{ }^{192} \mathrm{Os}$ (solid line) and ${ }^{40} \mathrm{Ca}+{ }^{194} \mathrm{Pt}$ (dashed line). 


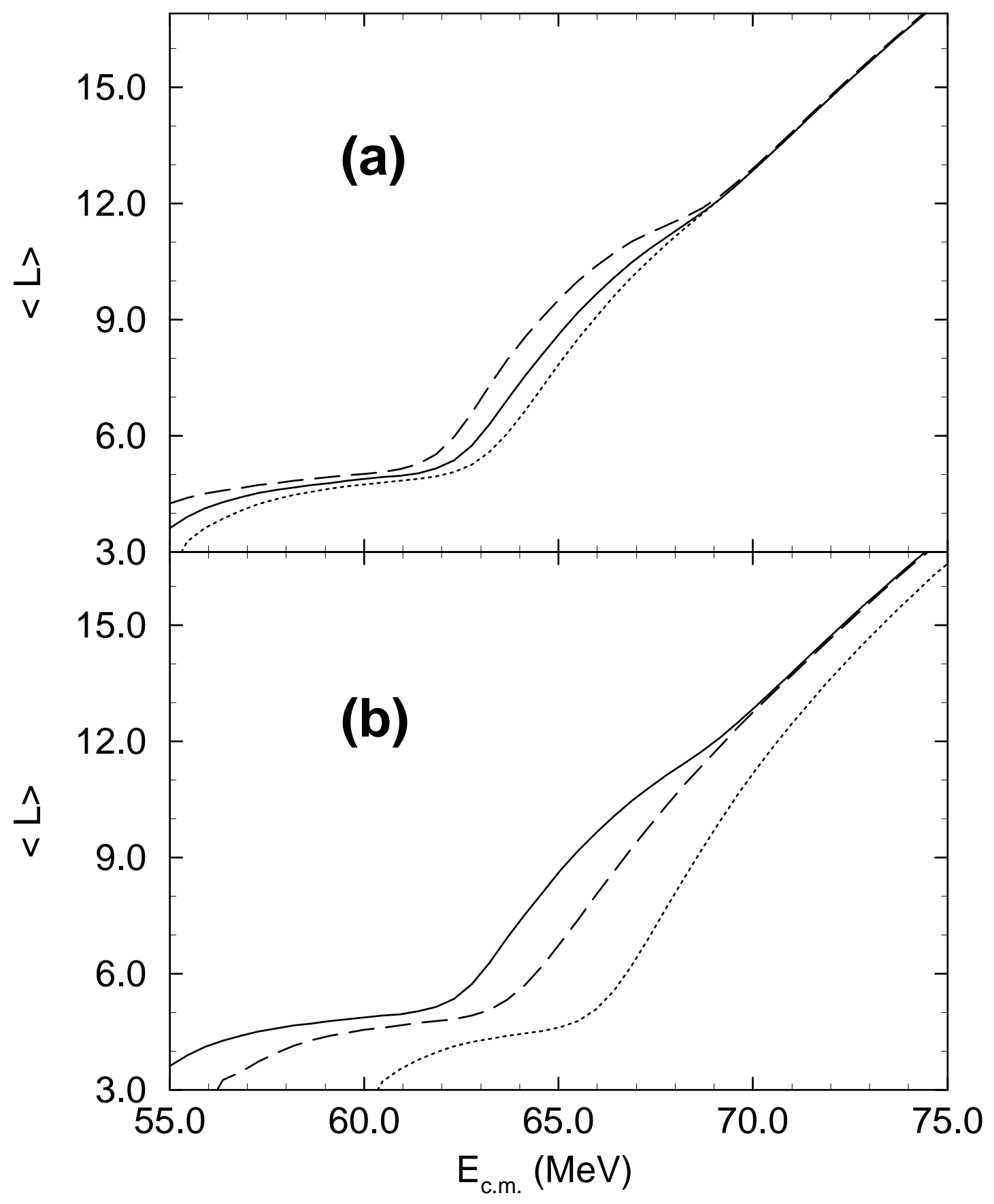




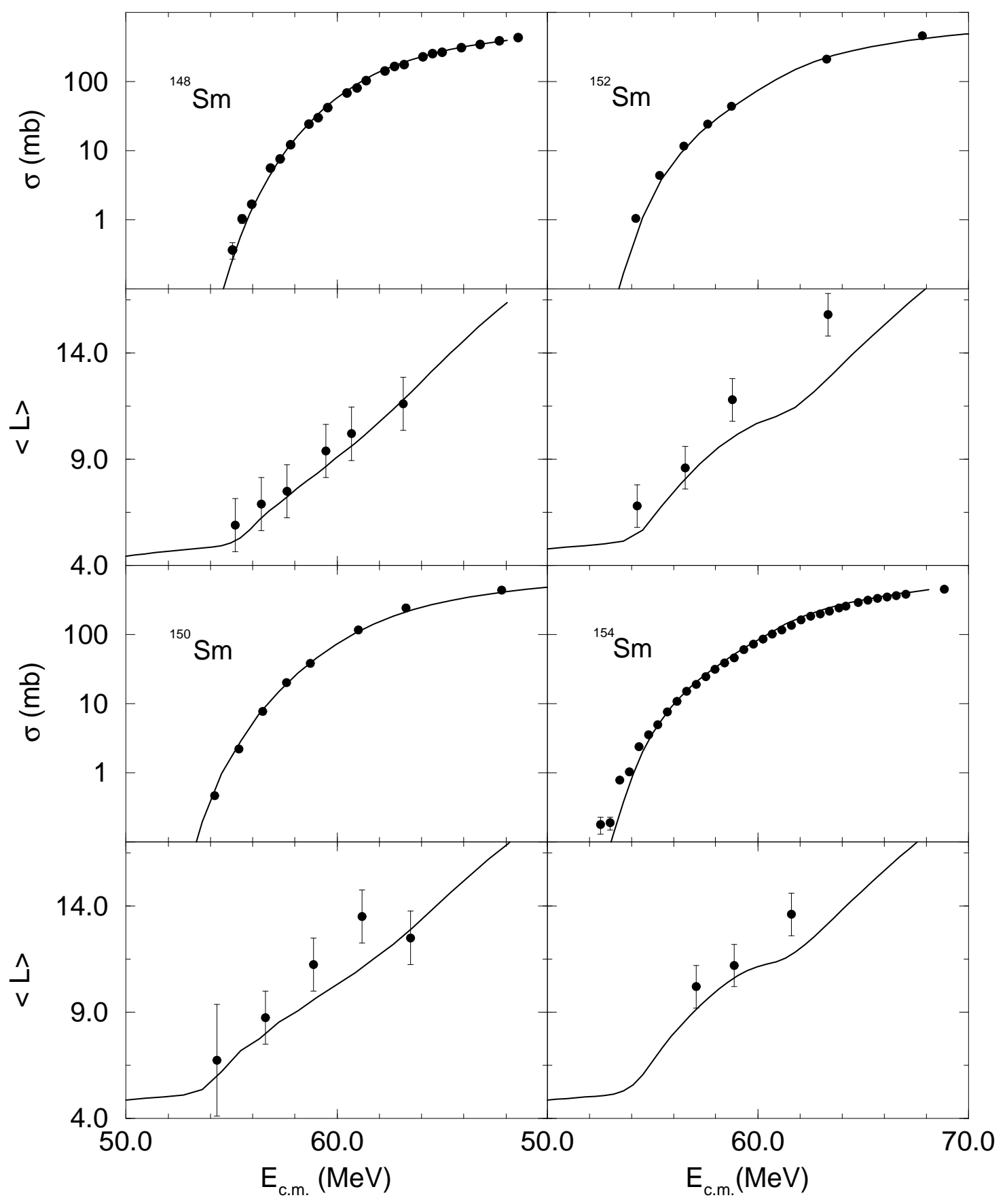



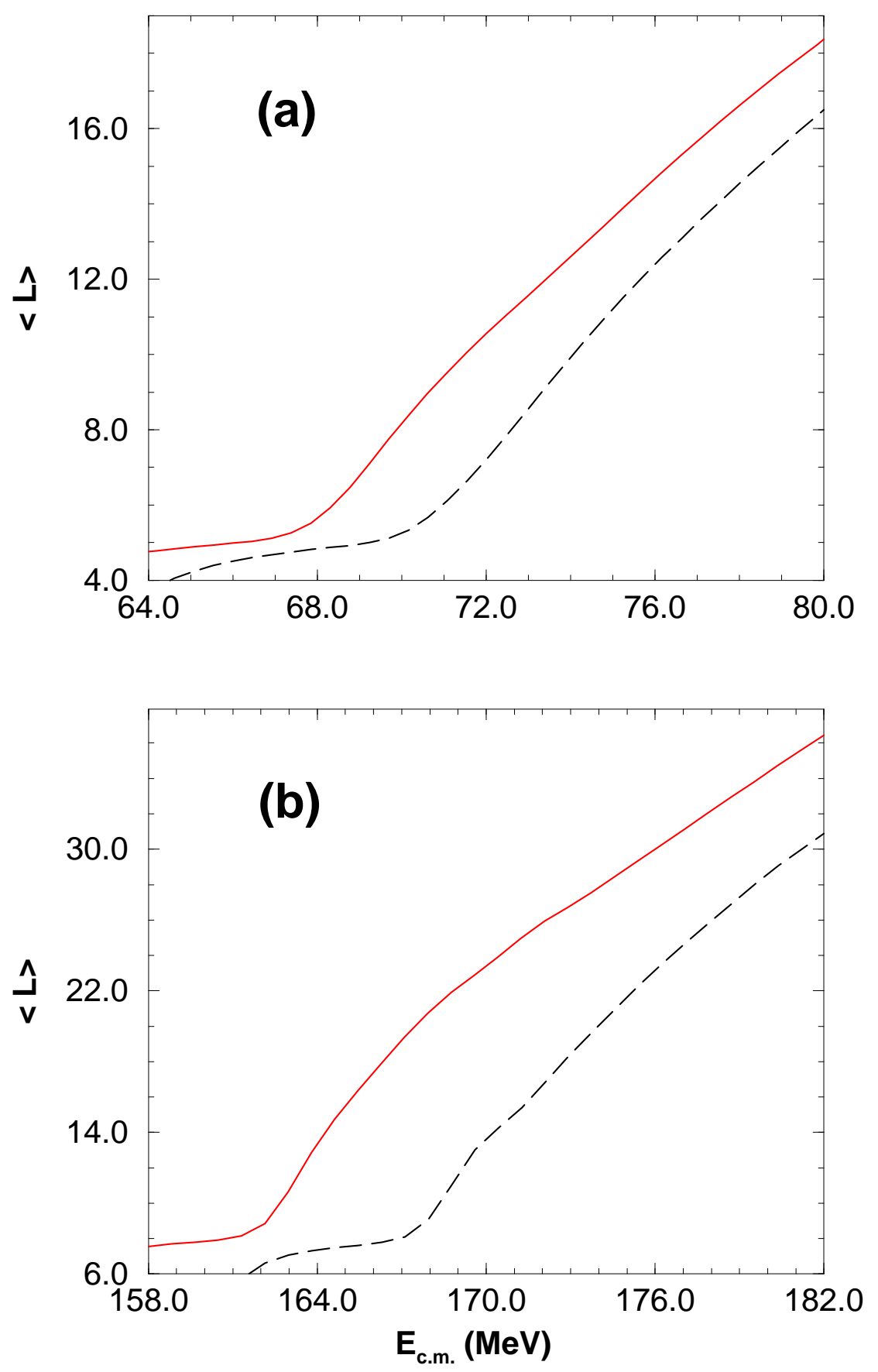